\title{
Antiprotons for Nuclear Structure Research
}

\author{
H. Lenske \\ Institut für Theoretische Physik, U. Giessen, \\ Heinrich-Buff-Ring 16, D-35392 Giessen
}

\begin{abstract}
The interaction of antiprotons with nuclei is investigated in a model using the free space $\bar{p} N$ T-matrix and nuclear densities from HFB calculations. Elastic scattering and annihilation reactions are used as probes for nuclear sizes of exotic nuclei. The annihilation cross sections are related to the nuclear root-mean-square radii by a scaling law. By detecting a pion ejected from the annihilation zone in coincidence with the final A-1 system the the annihilation process can be localized spatially and used to to extract specific information on the nuclear wave function.
\end{abstract}

\section{Introduction}

A central question of modern nuclear structure physics is to understand the evolution of nuclear shapes and sizes from stable to $\beta$ - unstable nuclei [1-4]. Experimentally, the properties of neutron-rich exotic nuclei are investigated by a large variety of methods [3-6]. An interesting alternative to the more traditional methods are investigations of radii and density distributions by antiprotons. Hitherto, the experiments have been performed with secondary antiproton beams on stable nuclei in fixed target geometry and standard kinematics. For stable nuclei, such experiments have been done at BNL, e.g. [7,8] and LEAR, e.g. [9-12]. In particular, anti-protonic atoms have been a major source of information on the tails of nuclear density distributions of stable nuclei, e.g. [11,13-16]. Obviously, another approach is needed when using antiprotons for reactions on short-lived isotopes. These nuclei by themselves are available only as secondary beams, produced either by fragmentation or isotope separation on line. A solution dissolving these conflicting conditions is to perform the measurements in colliding beam geometry. Such a setup was proposed in [17]. It will become feasible with the meanwhile approved FAIR facility a GSI [18]. The FAIR plans include as central components $\bar{p}$ production and accumulation facilities. For nuclear structure research the New Experimental Storage Ring (NESR) for short-lived nuclides including an intersecting electron accelerator for colliding beam experiments will be available. As discussed in the Antiproton-Ion-Collider (AIC) proposal [19], the NESR/ $e^{-}$setup can be converted into a $\bar{p} A$ collider facility.

Here, we investigate theoretically the use of antiprotons as probes for nuclear sizes of neutronrich nuclei, as proposed in $[2,17]$. The physics of $\bar{p} A$ interactions is by itself an interesting topic for nuclear and hadron physics. Here we concentrate on the use of $\bar{p}+A$ scattering for studies of neutron skins. For similar studies on stable nuclei see [7] and [9-12]. Here, we take advantage of the strong $\bar{p} A$ absorption for nuclear structure investigations without paying particular attention to the hadron physics aspects, except for utilizing pion production as an additional signature of the annihilation process.

The basic features of the approach are discussed in section 2 with special emphasis on the relation of the annihilation of high energy antiprotons on nuclei to the extraction of structure information. Exclusive pion production in $\bar{p} A$ annihilation reactions is discussed in section 4 . A summary and an outlook are given in section 5 .

\section{Antiproton-Nucleus Interactions}

From various experimental studies, starting as early as in the later 1950's [20], at least the on-shell (s-channel) behavior of the $\bar{p} N$ T-matrix is sufficiently well known in the energy region of interest. 


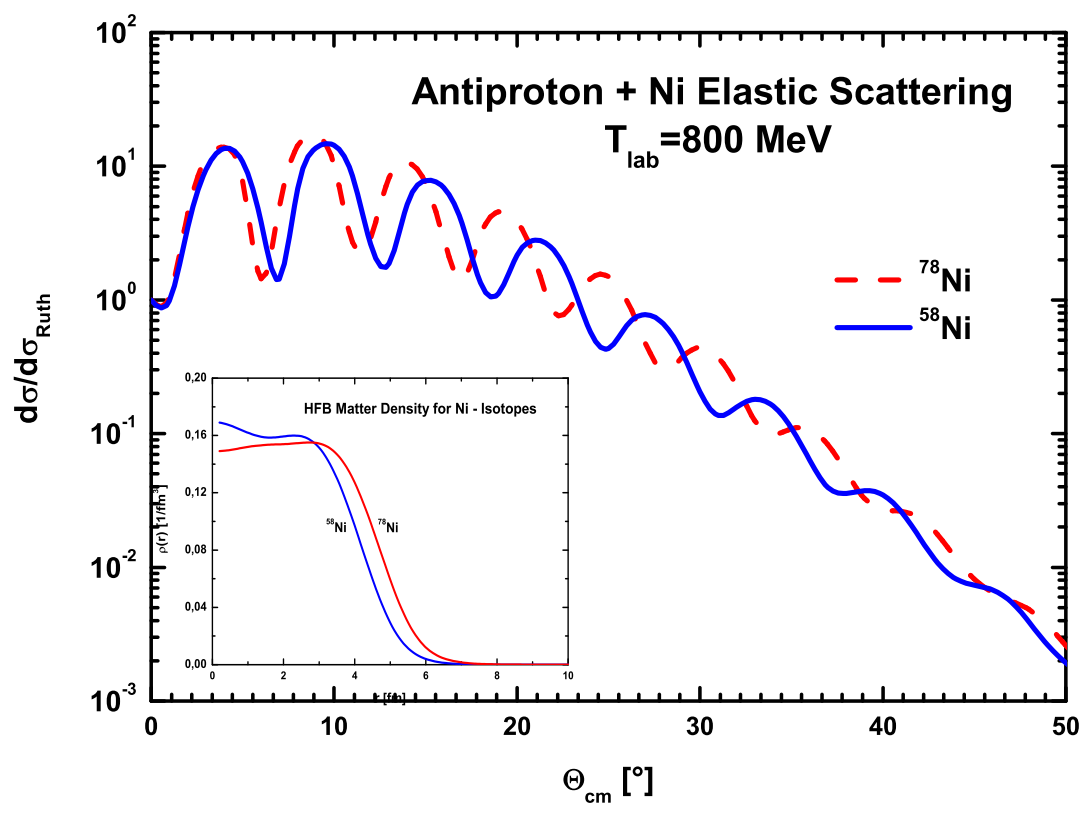

Fig. 1: Angular distributions for elastic scattering of antiprotons on a ${ }^{58} \mathrm{Ni}$ (full line) and a ${ }^{78} \mathrm{Ni}$ (dashed line) target nucleus at an incident energy of $T_{L a b}=800 \mathrm{MeV}$. The cross sections are normalized to the Rutherford cross section. The HFB ground state density distributions for the two nuclei are shown in the insert.

Naturally, the purely central spin-independent parts are known the best but some information on the spin-orbit parts is also available. The most significant property of the $\bar{p} N$ interaction is the dominance of absorptive components.

The origin of the $\bar{p} N$ interaction is still not well understood. Symmetry arguments based on Gparity do not agree with the observed properties of $\bar{p} A$ interactions and the derived empirical potentials, e.g. [16]. Hadronic models are in use which describe the annihilation process by effective, but microscopically unresolved, vertices. A first successful attempt in this direction was the work of the Paris group [21], using their G-parity transformed NN-potential supplemented by a purely phenomenological imaginary part. Similar ideas were applied shortly after by Timmers et al. [22]. That work is based on the G-parity transformed Nijmwegen NN-potential but the annihilation channels are simulated by explicit, although schematic and strongly simplified, coupled channels calculations.

Attempts to derive the $\bar{p} N$ interactions microscopically from the valence quark structure are only partly successful. In a quark-antiquark annihilation model Niskanen and Green [23] have derived some time ago a purely imaginary local $\bar{p} N$ potential. This so-called Ueda potential is given in coordinate space in terms of Gaussian form factors for each the central and the spin-orbit component. A possible reason for the deviations from standard $\bar{p} N$ interaction models could be due to strong dispersive contributions by dynamically induced interactions from higher order couplings to the annihilation channels.

Also $\bar{p} A$ interactions have been studied by various groups. Theoretical approaches based on microscopic descriptions of nuclear structure and elementary $\bar{p} N$ interactions have been used e.g. in $[24,25]$ to formulate a folding model following closely the successful approach used for $p A$ scattering [26]. The folding approach is especially well suited for systematic investigations of nuclear structure effects as it is the purpose of the present work. The result of the folding calculation is a complex optical poten- 


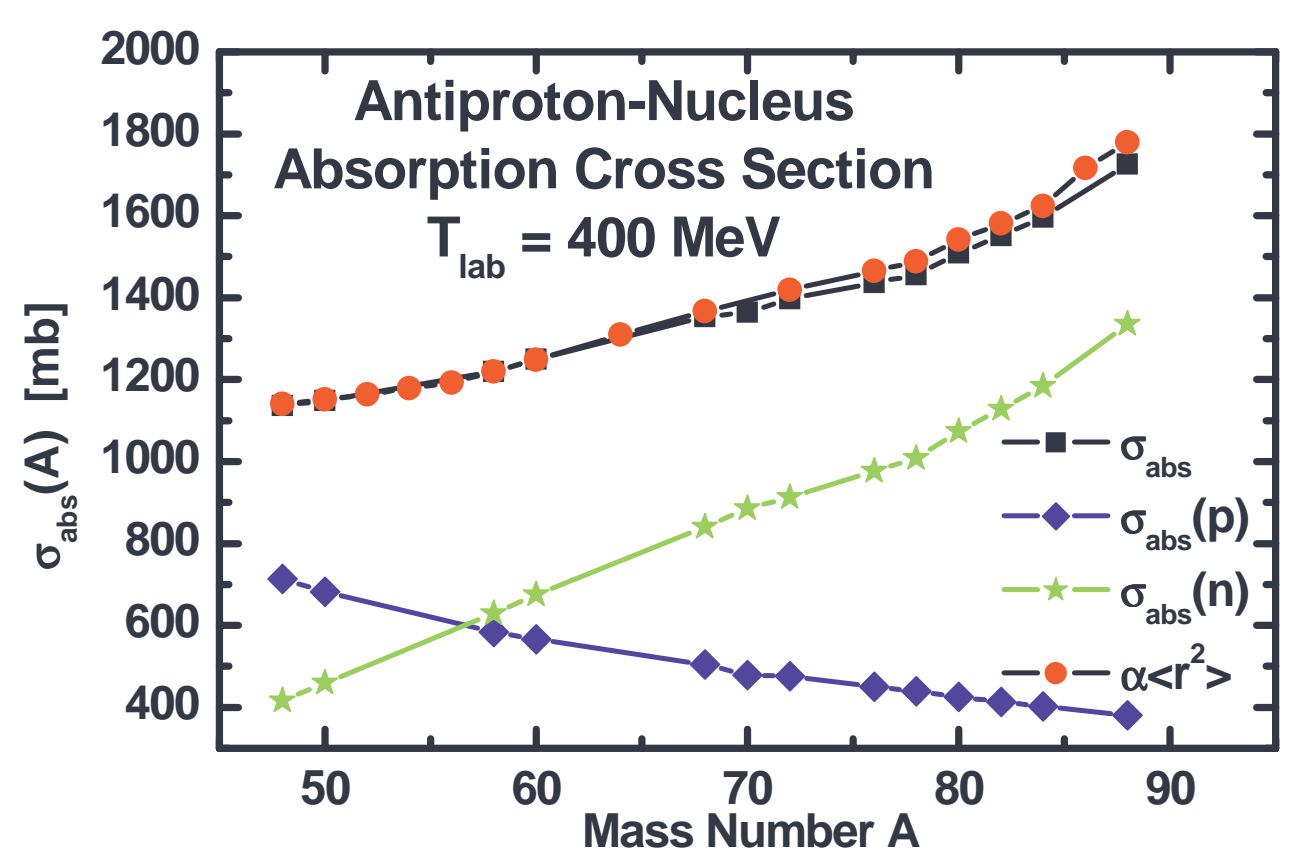

Fig. 2: Absorption cross sections (circles) for antiproton annihilation on Ni-isotopes at $T_{L a b}=400 \mathrm{MeV}$ are compared to the rms-radii of the nuclear matter densities (squares). The rms-radii are normalized to $\sigma_{a b s}$ for ${ }^{58} \mathrm{Ni}$. Also shown are the partial cross sections for absorption on the target neutrons and protons. For more details see [2].

tial $U_{\text {opt }}=U_{c}+V+i W$ with real and imaginary parts $V$ and $W$, respectively, and including also the Coulomb potential $U_{c}$. All pieces of the potential are calculated by folding the elementary short range strong and long range electromagnetic NN interactions with microscopic nucleonic HFB mass and charge charge densities. In the impulse or $t \rho$ approximation the coordinate space $\bar{p} A$ optical potential at energy $T_{L a b}$ is obtained as,

$$
U_{o p t}(\mathbf{r})=\sum_{N=p, n} \int \frac{d^{3} q}{(2 \pi)^{3}} \rho_{N}(q) t_{\bar{p} N}\left(T_{L a b}, q^{2}\right) e^{i \mathbf{q} \cdot \mathbf{r}}
$$

with the elementary $\bar{p} N$ momentum space T-matrix $t_{\bar{p} N}$ and the Fourier transforms $\rho_{p, n}(q)$ of the proton and neutron ground state densities. As the densities also the folding potential is additive in the partial proton and neutron contributions. Hence, we find $U_{o p t}=U_{o p t}^{(p)}+U_{o p t}^{(n)}$. As in free $\bar{p} N$ scattering the potentials are dominated by the strong imaginary parts.

In structure, eq. 1 resembles the expressions for $N-A$ nucleon-nucleus folding potentials, e.g. [26]. However, an obvious difference is that in the $\bar{p} A$ case anti-symmetrization effects are absent. Therefore, the exchange potentials, giving rise to non-local components in the $N-A$ potentials already on the tadpole level, do not contribute here. In that sense, the antiproton-nucleus interaction is simpler. But one has to be aware that the dispersive polarization potentials, induced by the annihilation processes, will contribute energy and momentum dependent parts of a specific structure. As in the general theory of the optical model [26] the projector technique may be used to derive the self-energy in the P-space, i.e. the Hilbert-space containing only the elastic $\bar{p} A$ scattering channel. As usual, the Q-space contains the whole multitude of non-elastic reaction channels, given mainly by the annihilation channels $|\bar{p} A\rangle \rightarrow$ $\left|k(h \bar{h}) A_{n}\right\rangle$ consisting of a residual target-like nucleus $A_{n}$ with $A-n$ nucleons and a number of hadronantihadron pairs $h \bar{h}$, including both mesonic and baryonic particles. At high energies, as considered here, we can expect that the annihilation channels will continue to dominate also in the $\bar{p} A$ case.

$$
\mathcal{U}_{p o l}(\mathbf{q} \mid \sqrt{s})=\left\langle\bar{p} A\left|t_{\bar{p} N}\right| G_{Q Q}(\sqrt{s}) \mid \bar{p} A\right\rangle
$$


where $G_{Q Q}$ is the Q-space Green function. The available energy in the center of mass frame is given by the Mandelstam variable $\sqrt{s}$. The momentum transfer is denoted by q. With the reasonable assumption that the T-matrix is dominated by two-body processes the channels producing the largest contributions will be those where the annihilation takes place on a single nucleon, leaving the $A-1$ nucleus in it's ground state. The energy released in the $\bar{p} N$ annihilation is forming a resonance state $|R\rangle$ of about a mass $M_{R} \sim 2 M_{N}$. Since at this stage we are not interested in resolving the spectral structure of that compound state (see e.g. [27]) but are only interested in the total intermediate yield and the decay back into the initial $|\bar{p} A\rangle$ configuration we treat the intermediate Q-space states in a spectator-like approximation, $\left|Q_{\alpha}\right\rangle=\left|R_{\alpha}\right\rangle|A-1\rangle_{\alpha}$. Hence, with respect to the initial $\bar{p} A$ channel, the intermediate states resemble a $N^{-1} R$ configuration and the polarization tensor, eq. 2, describes a self-energy of one hole-two particle $(1 h-2 p)$ character where the $|R\rangle=|\bar{p} N\rangle$ state is a strongly correlated configuration carrying bosonic quantum numbers. In fact, on the quite different scale of nuclear structure physics similar phenomena are welknown, namely the coupling of a hole (or particle) state to a strongly collective phonon-excitation of the Fermi-sea. In that language, eq. 2 may be considered as a $1 h-1 p 1 \bar{p}$ configuration with a strongly correlated $p \bar{p}$ component. At present, calculations utilizing this picture are in preparation.

For the time being, we use empirical input for for elementary $\bar{p} N$ T-matrix. The $\bar{p} A$ elastic scattering amplitudes and cross sections are obtained by solving a wave equation with the folding potentials, eq. 1 , and relativistic kinematics. The elastic angular distributions predicted for the scattering of antiprotons on ${ }^{58} \mathrm{Ni}$ and ${ }^{78} \mathrm{Ni}$ at $T_{L a b}=800 \mathrm{MeV}$ displayed together with the target mass densities in Fig. 2, show an interesting dependence on the nuclear density distributions. The larger extension of ${ }^{78} \mathrm{Ni}$ and changes in the surface properties due to the neutron skin lead to a quite different diffraction pattern clearly distinguishable from the results for the stable ${ }^{58} \mathrm{Ni}$ isotope. Hence, already the observation of elastic scattering cross sections will provide important information at least on the target size.

An interesting relation is obtained from the quantum mechanical continuity equation

$$
\sigma_{a b s}=\sum_{\ell j} \sigma_{a b s}(\ell j)=-\frac{2 \widetilde{m}}{k \hbar^{2}} \frac{1}{2 s+1} \operatorname{tr}_{s} \int d^{3} r \Psi_{s}^{(+) \dagger}(\mathbf{k}, \mathbf{r}) W(\mathbf{r}) \Psi_{s}^{(+)}(\mathbf{k}, \mathbf{r})
$$

expressing $\sigma_{a b s}$ directly in terms of the absorptive potential $W(\mathbf{r})=\Im U_{\text {opt }}(\mathbf{r})$. Since $W=W_{p}+W_{n}$ we conclude that $\sigma_{a b s}=\sigma_{a b s}^{(p)}+\sigma_{a b s}^{(n)}$ is additive in the proton and neutron partial contributions, describing the annihilation of the incoming $\bar{p}$ on the target protons and neutrons, respectively. This relation enables us to relate $\sigma_{a b s}$ to the rms radius of the target nucleus [2].

\section{Investigations of Nuclear Radii by $\bar{p}$ Nucleus Interactions}

\section{1 $\bar{p}$ Absorption on Ni-Isotopes}

The target ground states are described by HFB calculations in the DME approach [28]. We find a very satisfactory overall agreement of the theoretical and the measured binding energies on the level of 5\% and the known rms radii. The cross sections for $\bar{p}$ annihilation on ${ }^{48-88} \mathrm{Ni}$ show a striking similarity the mass dependence of the rms radii, at least at energies above $T_{L a b}>50 \mathrm{MeV}$ [2]. In order to understand the mechanism behind this correlation the absorption cross sections at $\mathrm{T}_{L a b}=400 \mathrm{MeV}$ are compared to the nuclear rms-radii in Fig. 2. We realize that the slopes of $\sigma_{a b s}$ and the rms radii are in almost exact agreement, indistinguishable at the scale of the figure. We find this behavior at all energies in an universal manner. Hence, we conclude that antiproton annihilation is an appropriate probe for nuclear sizes.

\subsection{Screening Effects in Neutron-rich Nuclei}

In eikonal approximation the scaling factor can, in fact, be calculated in closed form [2]. With the ansatz

$$
\sigma_{a b s}\left(A, T_{c m}\right)=\pi\left\langle r^{2}\right\rangle_{A} f\left(A, T_{c m}\right)
$$




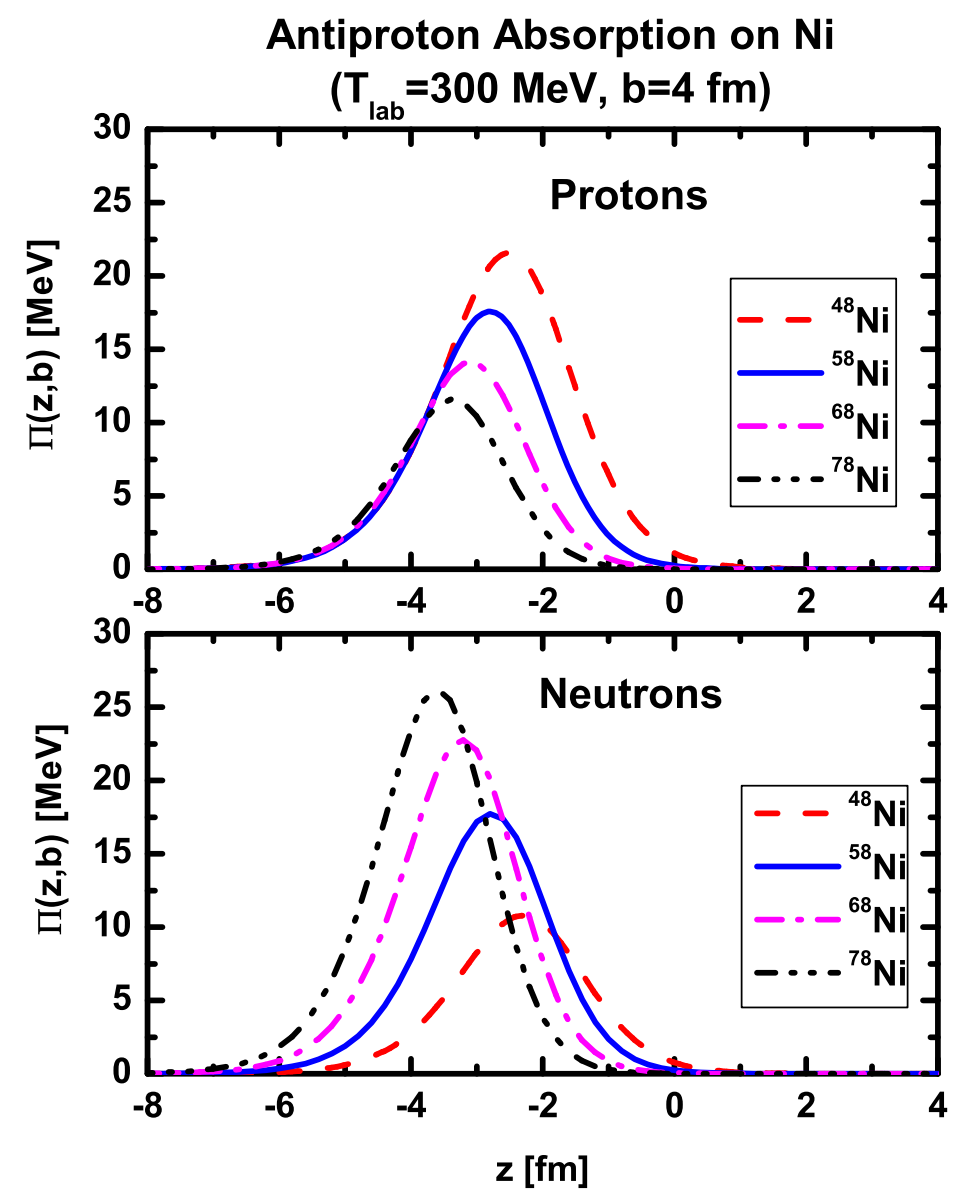

Fig. 3: Absorption of the incoming antiproton along a trajectory at the peripheral impact parameter $b=4 \mathrm{fm}$. The absorption kernels, obtained from eikonal calculations at an incident energy of $T_{L a b}=300 \mathrm{MeV}$ [2], are displayed for ${ }^{48,58,68,78} \mathrm{Ni}$. The $\bar{p}$ beam is incoming from the left. Note the reversed behavior of the $\bar{p}$ absorption on protons and neutrons with increasing neutron excess.

the scaling function is found to be given by $f\left(A, T_{c m}\right) \sim t_{\bar{p} N} \rho_{A} / T_{L a b}$, where $\rho_{A}$ denotes the central target density [2].

The dynamics of $\bar{p} A$ annihilation is understood more clearly by considering the integrand of eq. 3 , which corresponds to an absorption kernel $\Pi(\vec{r})$ [2]. In Fig. 3 the kernel $\Pi(z, b)$ is displayed for a representative selection of $\mathrm{Ni}$ nuclei at the grazing value $b=4 \mathrm{fm}$. In ${ }^{48} \mathrm{Ni}$ the absorption occurs mainly on the protons which in that neutron-deficient nucleus are sticking out, forming a proton skin. In the stable $A=58$ isotope with an almost equal number of protons and neutrons and negligible excess density the antiprotons are equally well absorbed on both neutrons and protons. By further adding neutrons the absorption on the neutron excess density obviously is strongly increasing, overshooting the absorption on protons by a factor of about 2 towards ${ }^{78} \mathrm{Ni}$. Hence, in neutron-rich nuclei the outer layer of neutron matter forming the skin density screens the target protons by absorbing the incoming antiprotons before they reach the region of the much stronger bound and therefore spatially much stronger confined protons. Thus, we find an intriguing interplay between neutron skins and $\bar{p}$ annihilation which is causing the decrease of the partial proton cross sections seen in Fig. 3. 


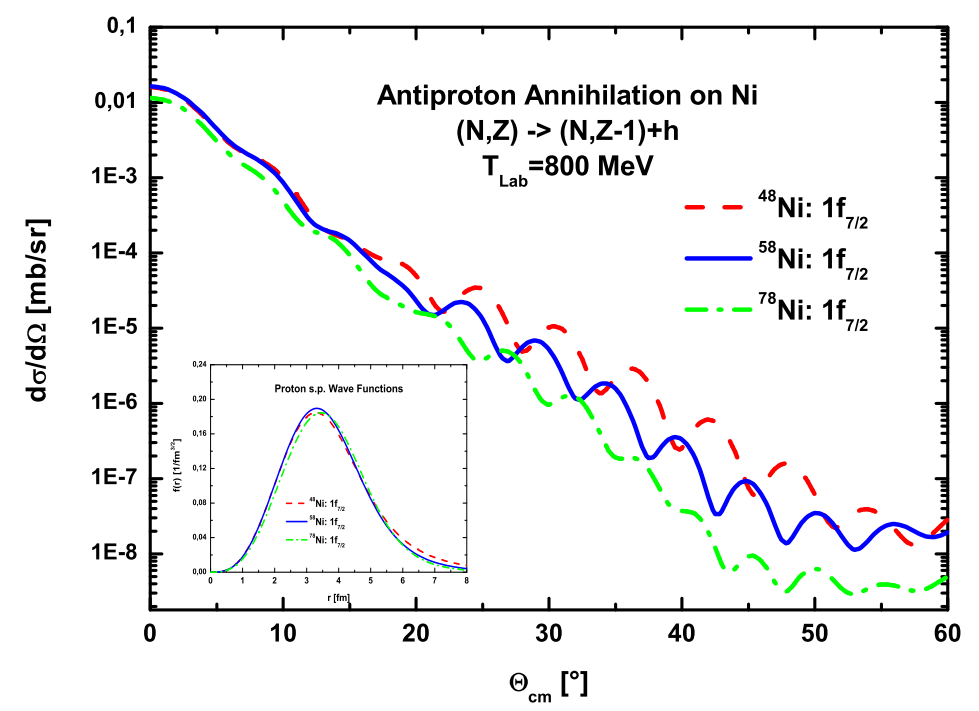

Fig. 4: Absorption of an incoming antiproton with an energy of $T_{L a b}=800 \mathrm{MeV}$ on a proton from the respective valence shell of a number of $\mathrm{Ni}$ isotopes. The radial proton wave functions are shown in the insert. Along the isotopic lines, the cross sections are slightly changing reflecting the corresponding changes in the $1 f_{7 / 2}$ proton valence wave functions which is mainly due to increase in the isovector potential.

\section{Exclusive Pion Production by Annihilation}

From the annihilation zone several pions will be ejected [29-31]. At given energy their number depends on the target mass, varying between 5 pions in $\bar{p} C$ to 2 pions in $\bar{p} U$ [29] due to rescattering and absorption processes. The pion production by annihilation takes place preferentially in the nuclear surface, hence the ejected pions carry important information on the spatial localization and the compositions of the medium in that area.

The more detailed picture of the annihilation process and the nuclear environment is taken advantage of by observing together with the emerging residual nucleus one or a few pions. By observation of the nucleus the final system is identified unanimously. The detection of the pion provides direct access to the momentum distribution of the nucleon on which the annihilation took place. The quantum mechanical cross section for coherent pion production on a nucleon in a nucleus has the structure

$$
d \sigma=\frac{d^{3} P_{\beta}}{(2 \pi)^{3}} \frac{d^{3} k_{\beta}}{(2 \pi)^{3}} \delta^{3}\left(\vec{P}_{\beta}-\vec{P}_{\alpha}\right) \sum_{n} \frac{d^{3} q_{n}}{(2 \pi)^{3}} \delta\left(T_{\beta}+T_{n}-T_{\alpha}\right)\left|M_{\alpha \beta}\left(\vec{k}_{\beta}, \vec{q}_{n}, \vec{k}_{\alpha}\right)\right|^{2}
$$

The total initial and final momenta are $P_{\alpha, \beta}$, asymptotic kinetic energies are denoted by $T_{\alpha, \beta, n}$ where momenta within the pion cloud are indicated by $q_{n}$. The invariant matrix element of the reaction can be written

$$
M_{\alpha \beta}=G_{0} \int d^{3} r_{\beta} d^{3} r_{\alpha} \chi_{\beta}^{(-) \dagger}\left(\vec{r}_{\beta}, \vec{k}_{\beta}\right) \varphi\left(\vec{r}_{n}\right) \chi_{\alpha}^{(+)}\left(\vec{r}_{\alpha}, \vec{k}_{\alpha}\right)
$$

where distorted waves $\chi_{\alpha, \beta}^{ \pm}$are used to describe the incident antiproton and the emitted pion cloud. $G_{0}$ is a coupling constant. Of primary interest for the present discussion is the appearance of the nucleon wave function $\varphi_{n}$ on which the $\bar{p} N \rightarrow n \pi$ takes place. From the above expression it is clearly seen that the cross section will depend very directly on the properties of the nuclear wave function, hence providing access to the spectroscopy and the nuclear form factor. In fact, by structure the matrix element resembles 


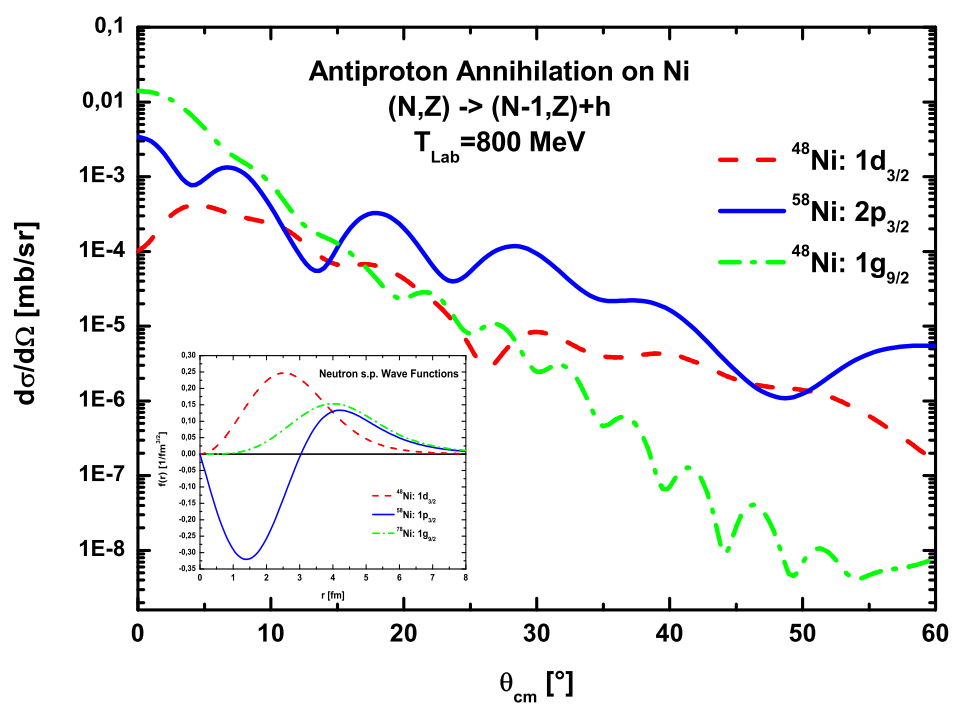

Fig. 5: Absorption of an incoming antiproton with an energy of $T_{L a b}=700 \mathrm{MeV}$ on a neutron from the respective valence shell of a number of $\mathrm{Ni}$ isotopes. The radial neutron wave functions are shown in the insert. In forward direction, the largest cross section is produced by the $g_{9 / 2}$ valence states of ${ }^{78} \mathrm{Ni}$, the second largest is due to pion production on the $2 p_{3 / 2}$ orbit in ${ }^{58} \mathrm{Ni}$.

the reaction amplitude of a breakup reaction, but providing additional and independent information on the momentum structure. This apparent sensitivity will also serve as an ideal tagger on the process and the spatial location of the annihilation event.

In Fig. 4 and Fig. 5 first results for the angular distribution produced by the $\bar{p}$ annihilation on a target proton and neutron, respectively, in the corresponding nuclear valence shells are displayed. For the neutron annihilation there is a pronounced dependence on the quantum numbers of the nucleonic states, most clearly visible at forward angles. The results of show that at forward angles the cross sections are increasing with the radial quantum number and the orbital angular momentum of the orbit at which the annihilation occurs. Additional spectral information is obtained from the diffraction structure at larger angles, i.e. momentum transfers.

\section{Conclusions}

Antiproton-nucleus scattering was investigated theoretically as a tool for measuring the sizes of neutron skins in exotic nuclei. Deriving the $\bar{p} A$ optical potentials microscopically in a folding approach from the elementary free space $\bar{p} N$ scattering amplitudes and HFB density distributions we have investigated reactions of high energetic antiprotons on stable and exotic nuclei. A selectivity of elastic scattering cross sections and the annihilation process on the target density is predicted. Our results show that both elastic and absorption cross section measurements already provide valuable new information on the densities of the target nuclei. A more detailed picture will emerge by taking into account also the pions ejected from the annihilation zone.

There are other exciting perspectives for antiprotons in nuclear structure research. Reaction channels like $\bar{p} p \rightarrow Y \bar{Y}$ implemented open baryonic strangeness into a nuclear system. They can be used to produce single and double $\Lambda$ hypernuclei, thus opening up an unique access to hypernuclear physics in neutronrich nuclei. 
Supported in part by DFG (contracts Le439/5, Le439/7, Le439/9), BMBF and GSI.

\section{References}

[1] H. Lenske et al., Progress in Particle and Nuclear Physics 59114 (2007).

[2] H. Lenske, P. Kienle, Phys. Lett. B647 82 (2007).

[3] N. Tsoneva, H. Lenske, Phys. Lett. B586 213 (2004).

[4] N. Tsoneva, H. Lenske, Phys. Rev. C77 024321 (2008).

[5] H. Lenske, G. Schrieder, Eur. Phys. J. A2 41 (1998).

[6] A. Krasznahorkay et al., Phys. Rev. Lett. 823216 (1999); A. Krasznahorkay et al., Proc. of the INPC 2001, Berkeley, Aug. 2001, ed. by E. Norman et al., p.751 (2001).

[7] W. M. Bugg et al., Phys. Rev. Lett. 31475 (1973).

[8] V. Ashford et al., Phys. Rev. C30 1080 (1984).

[9] S. Janouin et al., Nucl.Phys. A451 (1986) 541.

[10] G. Bruge et al., Phys. Lett. B169 14 (1986).

[11] P. Lubinski et al., Phys. Rev. Lett. 733216 (1994)x.

[12] S. Wycech, J. Skalski, R. Smolanczuk, J. Dobaczewski, J.R. Rook, Phys. Rev. C54 1832 (1996).

[13] P. Lubinks, J. Jastrzebski, A. Trzcinska, W. Kurcewicz, F.J. Hartmann, R. Schmidt, T. von Egidy, R. Smolanczuk, S. Wycech,, Phys. Rev. C57 2962 (1998).

[14] A. Trzinska, J. Jastrzebski, P. Lubinski, F.J. Hartmann, R. Schmidt, T. von Egidy, B. Klos, Phys. Rev Lett. 87082501 (2001).

[15] C.J. Batty, E. Friedman, A. Gal, Phys. Rep. 287385 (1997).

[16] E. Friedman, A. Gal, J. Mares, Nucl. Phys. A761 283 (2005).

[17] P.Kienle, NIMB 214193 (2004).

[18] Proposal for the GSI Future Project, http://www.gsi.de.

[19] Proposal for an Antiproton-Ion Collider at FAIR, The AIC Collaboration, GSI, Darmstadt 2005.

[20] T. Elioff, L. Agnew, O. Chamberlain, H.M. Steiner, C. Wiegand, T. Ypsilantis, Phys. Rev. 128869 (1962).

[21] J. Cote, M. Lacombe, B. Loiseau, B. Moussallam and R. Vinh-Mau, Phys. Rev. Lett. 481319 (1982).

[22] P.H. Timmers et al., Phys. Rev. D 291928 (1984).

[23] J.A. Niskanen, Anthony M. Green, Nucl.Phys. A404 (1983) 495.

[24] Tan Zhen-Qiangi and Gu Yun-Ting, J. Phys. G: Nucl. Part. Phys 151699 (1989).

[25] Zhang Yu-shun, Liu Ji-feng, B. A. Robson, and Li Yang-guo, Phys.Rev. C54 332 (1996).

[26] H. Feshbach, Theoretical Nuclear Physics. Volume 2: Nuclear Reactions,John Wiley and Sons Inc, New York, 1993.

[27] A. Reuber et al., Nucl. Phys. A608 243 (1996).

[28] F. Hofmann, H. Lenske, Phys. Rev. C57 2281 (1998).

[29] M.R. Clover et al., Phys. Rev. C26 2138 (1982).

[30] E. Hernandez, E. Oset, Nucl. Phys. A455 584 (1986).

[31] E. Hernandez, E. Oset, Phys.Lett. B181 207 (1986). 